\title{
2'-Deoxyguanosine Toxicity for $B$ and Mature T Lymphoid Cell Lines Is Mediated by Guanine Ribonucleotide Accumulation
}

\author{
Yechezkel Sidi and Beverly S. Mitchell \\ Simpson Memorial Research Institute, Department of Internal \\ Medicine, University of Michigan, Ann Arbor, Michigan 48109
}

bstract. Inherited deficiency of the enzyme purine nucleoside phosphorylase (PNP) results in selective and severe $T$ lymphocyte depletion which is mediated by its substrate, 2'-deoxyguanosine. This observation provides a rationale for the use of PNP inhibitors as selective $T$ cell immunosuppressive agents. We have studied the relative effects of the PNP inhibitor 8-aminoguanosine on the metabolism and growth of lymphoid cell lines of $\mathrm{T}$ and $\mathrm{B}$ cell origin. We have found that 2'-deoxyguanosine toxicity for $\mathrm{T}$ lymphoblasts is markedly potentiated by 8 -aminoguanosine and is mediated by the accumulation of deoxyguanosine triphosphate. In contrast, the growth of $\mathrm{T}_{4}{ }^{+}$mature $\mathrm{T}$ cell lines and B lymphoblast cell lines is inhibited by somewhat higher concentrations of 2 -deoxyguanosine (ID $_{50}$ 20 and $18 \mu \mathrm{M}$, respectively) in the presence of 8aminoguanosine without an increase in deoxyguanosine triphosphate levels. Cytotoxicity correlates instead with a three- to fivefold increase in guanosine triphosphate (GTP) levels after $24 \mathrm{~h}$. Accumulation of GTP and growth inhibition also result from exposure to guanosine, but not to guanine at equimolar concentrations. B lymphoblasts which are deficient in the purine salvage enzyme hypoxanthine guanine phosphoribosyltransferase are completely resistant to 2 '-deoxyguanosine or guanosine concentrations up to $800 \mu \mathrm{M}$ and do not demonstrate an increase in GTP levels. Growth inhibition and GTP accumulation are prevented by hypoxanthine or adenine, but not by 2 -deoxycytidine. 8-Aminoguano-

Dr. Sidi's current address is Department of Medicine D, Beilinson Medical Center, Petah tikva, Israel. Address correspondence to Dr. Mitchell.

Received for publication 31 January 1984 and in revised form 12 July 1984.

\section{J. Clin. Invest.}

(c) The American Society for Clinical Investigation, Inc. 0021-9738/84/11/1640/09 \$1.00

Volume 74, November 1984, 1640-1648 sine appears to effectively inhibit extracellular PNP activity; thus, it prolongs the extracellular half-life of 2 'deoxyguanosine and guanosine, but does not completely inhibit intracellular PNP activity in these lymphoid cells. As a result, 2'-deoxyguanosine and guanosine are phosphorolyzed and actively salvaged within the cell, accounting for the accumulation of GTP. Partial inhibition of PNP activity in vivo, therefore, may lead to nonselective cellular toxicity by a mechanism independent of dGTP accumulation.

\section{Introduction}

Inherited deficiency of the enzyme purine nucleoside phosphorylase (PNP) ${ }^{1}$ results in a severe depletion of T lymphocytes and loss of cellular immunity $(1,2)$. The immunodeficiency disease appears to result from the selective metabolism of the PNP substrate, 2'-deoxyguanosine, to the deoxyribonucleotide, deoxyguanosine triphosphate (dGTP), in the T cells of affected individuals (3). This hypothesis has been given added credence by the observation that cultured human lymphoblasts of $T$ cell origin accumulate large amounts of dGTP when exposed to 2 -deoxyguanosine in vitro $(4,5)$. dGTP inhibits the enzyme ribonucleotide reductase, which catalyzes the reduction of nucleoside diphosphates to their corresponding 2'-deoxy derivatives. Thus, accumulation of dGTP leads to depletion of other deoxynucleoside triphosphates, inhibition of DNA synthesis, and cell death $(6,7)$. Human B lymphoblasts and mature peripheral blood lymphocytes are both relatively resistant to the toxic effects of 2'-deoxyguanosine alone and do not accumulate significant amounts of dGTP under in vitro culture conditions (8-10).

As a consequence of these observations, there has been considerable interest in the development of PNP inhibitors as potentially selective $\mathrm{T}$ cell cytotoxic agents. We have previously reported that the guanosine analogue, 8-aminoguanosine, is a

1. Abbreviations used in this paper: dGTP, deoxyguanosine triphosphate; HPRT, hypoxanthine guanine phosphoribosyltransferase; GDP, guanosine diphosphate; GMP, guanosine monophosphate; GTP, guanosine triphosphate; PNP, purine nucleoside phosphorylase; PP-ribose-P, 5phosphoribosyl 1-pyrophosphate. 
competitive inhibitor of PNP which markedly potentiates both the toxicity of $2^{\prime}$-deoxyguanosine and the accumulation of dGTP in T lymphoblasts (9). We now have evidence that 8-aminoguanosine also markedly enhances the toxic effects of 2'-deoxyguanosine for cultured $B$ lymphoblasts and mature $T$ cell lines. The inhibition of growth in these cells is mediated not by dGTP, but by the accumulation of guanine ribonucleotides.

\section{Methods}

Materials. 2'-Deoxyguanosine, guanosine, guanine, hypoxanthine, adenine, and $2^{\prime}$-deoxycytidine were purchased from Sigma Chemical Co. (St. Louis, MO). 8-Aminoguanosine was a gift of Dr. L. Townsend, Department of Medicinal Chemistry, University of Michigan, Ann Arbor, MI. Horse serum and RPMI 1640 medium were purchased from Gibco Laboratories (Grand Island, NY).

Cell lines. Human T lymphoblast lines Molt 4 and RPMI 8402 were derived from patients with $T$ cell acute lymphoblastic leukemia and obtained from HEM Research, Rockville, MD. The B lymphoblast lines GM 558, GM 1899 (hypoxanthine guanine phosphoribosyltransferase [HPRT ${ }^{-}$]), and GM $2292\left(\mathrm{HPRT}^{-}\right)$were obtained from the Human Genetic Mutant Cell Repository, Bethesda, MD. Line MGL 8 was obtained from the laboratory of Dr. John Littlefield, Johns Hopkins University, Baltimore, MD. The mature T cell lines HUT 78 and HUT 102 were obtained from Dr. Paul Bunn at the National Cancer Institute and have been described previously $(11,12)$. These cells were originally obtained from the peripheral blood or lymph node of patients with cutaneous $\mathrm{T}$ cell lymphomas and have surface antigens reactive with OKT4 monoclonal antibodies. The HUT 102 line is known to possess human $T$ cell leukemia virus (12). All cell lines were propagated in RPMI- 1640 medium with $10 \%$ horse serum at $37^{\circ} \mathrm{C}$ in $5 \% \mathrm{CO}_{2}$.

Cell growth experiments. Exponentially growing cells were suspended at a concentration of $2 \times 10^{5}$ cells $/ \mathrm{ml}$ in RPMI- 1640 medium, $10 \%$ horse serum, and the additives indicated in a final volume of $100 \mu \mathrm{l}$ in flat-bottom microtiter plates (Corning Medical and Scientific, Corning NY). Each experiment was performed in triplicate and control growth was exponential over $96 \mathrm{~h}$. Cell counts were performed at $72 \mathrm{~h}$ by hemocytometer, using trypan blue dye exclusion to identify viable cells. Counts were expressed as viable cells per milliliter and related to the growth of the cells designated as controls.

Nucleotide pool measurements. Cell incubations were performed at the final concentration of $2 \times 10^{5}$ cells/ml in RPMI-1640 medium with $10 \%$ horse serum and the additives indicated. 8-Aminoguanosine was added $30 \mathrm{~min}$ before the addition of other additives. Following the incubations, cells were sedimented at $400 \mathrm{~g}$, the supernatants carefully aspirated, and the cells extracted in $1 \mathrm{ml} 60 \%$ methanol at $-20^{\circ} \mathrm{C}$ for $24 \mathrm{~h}$. The percentage of viable cells was determined before each extraction and $0.5-2 \times 10^{7}$ cells were extracted for each determination. Ribo- and deoxyribonucleotides were separated by high pressure liquid chromatography on a Partisil-10 SAX anion-exchange column (Whatman Laboratory Products, Inc., Clifton, NJ), using an ammonium phosphate elution gradient $(0.3-0.45 \mathrm{M}, \mathrm{pH}$ 3.3-3.6) over $35 \mathrm{~min}$ at a flow rate of $2 \mathrm{ml} / \mathrm{min}$. Eluted nucleotides were detected by their absorbance at $254 \mathrm{~nm}$ and quantitated by comparison to calibration curves of nanomole amounts of pure standards. This method resulted in the complete separation of cytidine triphosphate, uridine triphosphate, ATP, deoxyadenosine triphosphate, GTP, and dGTP.
dGTP levels in unincubated T lymphoblasts and in B lymphoblast and mature $T$ cell lines required quantitation by the more sensitive DNA polymerase assay, which was performed as previously described (9).

Extracellular 2'-deoxyguanosine or guanosine levels were measured in cell culture supernates which were deproteinized by the method of Koller et al. (13). $0.96 \mathrm{ml} \mathrm{H}_{2} \mathrm{O}$ and $0.4 \mathrm{ml}$ of $0.15 \mathrm{M} \mathrm{Ba}(\mathrm{OH})_{2}$ were added to $1 \mathrm{ml}$ of supernate. After $2 \mathrm{~min}, 0.64 \mathrm{ml}$ of $0.15 \mathrm{M} \mathrm{ZnSO}_{4}$ was added, and the resultant sediment was discarded. The supernate was run on a reverse phase C-18 $\mu$ Bondapak column (Whatman Associates, Milford, MA) at a flow rate of $2 \mathrm{ml} / \mathrm{min}$ and eluted with $0.05 \mathrm{M} \mathrm{K}_{2} \mathrm{HPO}_{4}$ and $5 \%$ methanol, $\mathrm{pH}$ 2.6. The recovery of 2 -deoxyguanosine and guanosine by this procedure consistently ranged between 55 and $62 \%$.

\section{Results}

Cell growth experiments. The effects of 2'-deoxyguanosine and guanosine in the absence and presence of 8-aminoguanosine on the growth of $\mathrm{T}$ lymphoblasts, B lymphoblasts, and mature $T$ cell lines are summarized in Table $I$ and representative growth curves in the presence of 8-aminoguanosine are shown in Fig. 1. 8-Aminoguanosine at a concentration of $100 \mu \mathrm{M}$ markedly potentiates 2 -deoxyguanosine toxicity for $T$ lymphoblasts, as has been previously described (9). In addition, it enhances the toxicity of both $2^{\prime}$-deoxyguanosine and guanosine for B lymphoblasts and for mature T cell lines. B lymphoblasts deficient in the enzyme HPRT are completely resistant to 2 '-deoxyguanosine or guanosine concentrations up to $800 \mu \mathrm{M}$ in both the absence and presence of 8 -aminoguanosine. Fig. 1 demonstrates that, in the presence of 8-aminoguanosine, 2 -deoxyguanosine and guanosine have equivalent growth inhibitory effects on $B$ and mature $T$ cell lines, whereas $T$ lymphoblasts are both more sensitive to 2'-deoxyguanosine and more resistant to guanosine. Guanine inhibits the growth of $B$ and mature $T$ cell lines to a similar degree as 2'deoxyguanosine or guanosine alone, but guanine toxicity is not potentiated by 8 -aminoguanosine (Table I).

2 -Deoxycytidine at a concentration of $100 \mu \mathrm{M}$ markedly reduces the toxicity of 2'-deoxyguanosine and 8-aminoguanosine for T lymphoblasts (Fig. $2 A$ ), but has a far less marked rescuing effect on 2 -deoxyguanosine-mediated growth inhibition of B lymphoblasts (Fig. $2 \mathrm{~B}$ ) and mature T cells (Fig. $2 \mathrm{C}$ ). Uridine at concentrations up to $100 \mu \mathrm{M}$ does not prevent $2^{\prime}$ deoxyguanosine-induced growth inhibition of either B lymphoblasts or mature $\mathrm{T}$ cells (data not shown). In contrast, both adenine $(100 \mu \mathrm{M})$ and hypoxanthine $(100 \mu \mathrm{M})$ completely prevent the inhibition of growth in B lymphoblasts (Fig. $3 B$ ) and mature $\mathrm{T}$ cell lines (Fig. $3 C$ ), but have no effect on the toxicity of 2'-deoxyguanosine for T lymphoblasts (Fig. $3 \mathrm{~A}$ ).

Nucleotide pool determinations. The effects of incubations of the various cell lines with $100 \mu \mathrm{M}$ 8-aminoguanosine and $50 \mu \mathrm{M} 2$ '-deoxyguanosine on GTP and dGTP pools are shown in Fig. 4. T lymphoblasts did not accumulate any GTP, whereas $\mathrm{HPRT}^{+}$B lymphoblasts and mature $\mathrm{T}$ cell lines 
Table I. The Effect of 8-Aminoguanosine on 2'-Deoxyguanosine, Guanosine, and Guanine Toxicity for Lymphoid Cell Lines

\begin{tabular}{|c|c|c|c|c|c|c|c|}
\hline \multirow[b]{3}{*}{ Cell lines } & & \multicolumn{6}{|l|}{$\mathrm{ID}_{50}(\mu M)$} \\
\hline & & \multicolumn{2}{|c|}{ 2'-Deoxyguanosine } & \multicolumn{2}{|l|}{ Guanosine } & \multicolumn{2}{|l|}{ Guanine } \\
\hline & & $-8 \mathrm{AGuo}$ & $+8 \mathrm{AGuo}$ & -8 AGuo & $+8 \mathrm{AGuo}$ & $-8 \mathrm{AGuo}$ & $+8 \mathrm{AGuc}$ \\
\hline \multirow[t]{2}{*}{ T lymphoblasts } & Molt 4 & 20 & 1.3 & 210 & 152 & 350 & 400 \\
\hline & RPMI 8402 & 22 & 0.9 & 300 & 240 & ND & $\mathrm{ND}^{*}$ \\
\hline \multirow[t]{4}{*}{ B lymphoblasts } & MGL 8 & 60 & 18 & 160 & 12 & 160 & 150 \\
\hline & GM 558 & 160 & 17 & 250 & 14 & ND & ND \\
\hline & GM $2292\left(\mathrm{HPRT}^{-}\right)$ & $>800$ & $>800$ & $>800$ & $>800$ & $>600$ & $>600$ \\
\hline & GM $1899\left(\mathrm{HRPT}^{-}\right)$ & $>800$ & $>800$ & $>800$ & $>800$ & ND & ND \\
\hline \multirow{2}{*}{$\begin{array}{l}\text { Mature } T \\
\text { cells }\end{array}$} & HUT 78 & 75 & 20 & 80 & 15 & 200 & 150 \\
\hline & HUT 102 & 280 & 30 & 600 & 70 & ND & ND \\
\hline
\end{tabular}

Cell growth experiments were performed at a starting concentration of $2 \times 10^{5} \mathrm{cells} / \mathrm{ml}$ in RPMI- 1640 medium plus $10 \%$ horse serum in the absence or presence of $100 \mu \mathrm{M} 8$-aminoguanosine (8AGuo) and increasing concentrations of either 2'-deoxyguanosine, guanosine, or guanine. Each experiment was performed in triplicate and repeated on at least two occasions. The SEM on triplicate determinations was $<15 \%$. Values represent the mean concentrations of $2^{\prime}$-deoxyguanosine, guanosine, or guanine resulting in $50 \%$ inhibition of growth at $72 \mathrm{~h}$. ${ }^{*} \mathrm{ND}$, not done.

increased their GTP pools from three- to fivefold. HPRTdeficient B lymphoblasts did not accumulate any GTP under identical conditions. In contrast, $\mathrm{T}$ lymphoblasts accumulated large amounts of dGTP, as has been previously reported (9), whereas $B$ and mature $T$ cell lines were both resistant to dGTP accumulation.

The resistance of HPRT-deficient B lymphoblasts to both $2^{\prime}$-deoxyguanosine-mediated toxicity and GTP accumulation in the presence of 8-aminoguanosine led us to postulate that guanine ribonucleotide accumulation depended first upon the conversion of 2'-deoxyguanosine to guanine by PNP activity and, secondly, on the salvage of guanine to guanine nucleotides by HPRT activity (Fig. 5). The conversion of guanine to guanosine monophosphate (GMP) also requires 5-phosphoribosyl 1-pyrophosphate (PP-ribose-P) (Fig. 5). Since both adenine and hypoxanthine are also actively salvaged by phosphoribosylation and, hence, would compete with guanine for intracellular PP-ribose-P pools, we examined the effects of these compounds on intracellular GTP accumulation (Fig. 6). Both adenine $(100 \mu \mathrm{M})$ and hypoxanthine $(100 \mu \mathrm{M})$ completely prevented the GTP accumulation potentiated by 8-aminoguanosine at $24 \mathrm{~h}$. We, then, compared the effects of 2'deoxyguanosine in the absence and presence of 8-aminoguanosine with those of guanosine and guanine on GTP pools (Table II). 2'-Deoxy-guanosine and guanosine caused very similar elevations in GTP pools, which were markedly potentiated by 8 -aminoguanosine. Equimolar concentrations of guanine, on the other hand, did not increase GTP levels and the addition of 8-aminoguanosine had no enhancing effect.

To examine the mechanism by which 8 -aminoguanosine potentiates GTP accumulation, the levels of both GTP (Fig. 6 $A$ ) and extracellular 2'-deoxyguanosine (Fig. $6 \mathrm{~B}$ ) were moni- tored simultaneously over a 48-h culture of B lymphoblasts. In the absence of 8-aminoguanosine, GTP accumulated rapidly and reached a peak level of $4,000 \mathrm{pmol} / 10^{6}$ cells within $3 \mathrm{~h}$, with a return to base-line levels by $24 \mathrm{~h}$, while extracellular $2^{\prime}-$ deoxyguanosine concentrations dropped precipitously over a 12 -h period. The addition of 8 -aminoguanosine markedly prolonged the half-life of 2 -deoxyguanosine in the medium (Fig. $7 \mathrm{~B}$ ), and the intracellular GTP concentration remained elevated at a level more than three times the base-line concentration over the entire 48-h incubation period (Fig. $7 A$ ). Similar results were obtained using guanosine. 8-Aminoguanosine alone in concentrations up to $400 \mu \mathrm{M}$ did not cause change of GTP concentrations at any time point. However, in the presence of $50 \mu \mathrm{M} 2^{\prime}$-deoxyguanosine, increasing the 8-aminoguanosine concentration from $100 \mu \mathrm{M}$ to $400 \mu \mathrm{M}$ effectively prevented both the accumulation of GTP and inhibition of growth in B and mature T cell lines (Fig. 8). We conclude from these experiments that 8-aminoguanosine at lower concentrations competitively inhibits the phosphorolysis of 2'-deoxyguanosine and of guanosine by extracellular PNP activity, thereby prolonging the availability of the nucleosides for transport. 2'-Deoxyguanosine and guanosine are then cleaved to guanine by intracellular PNP activity and salvaged by HPRT. Higher concentrations of 8-aminoguanosine, on the other hand, more completely inhibit intracellular PNP activity, thereby preventing guanine ribonucleotide-related toxicity.

\section{Discussion}

We have established that the toxicity of 2'-deoxyguanosine for human B lymphoblast and mature $T$ cell lines is mediated by the accumulation of guanine ribonucleotides. The evidence to 

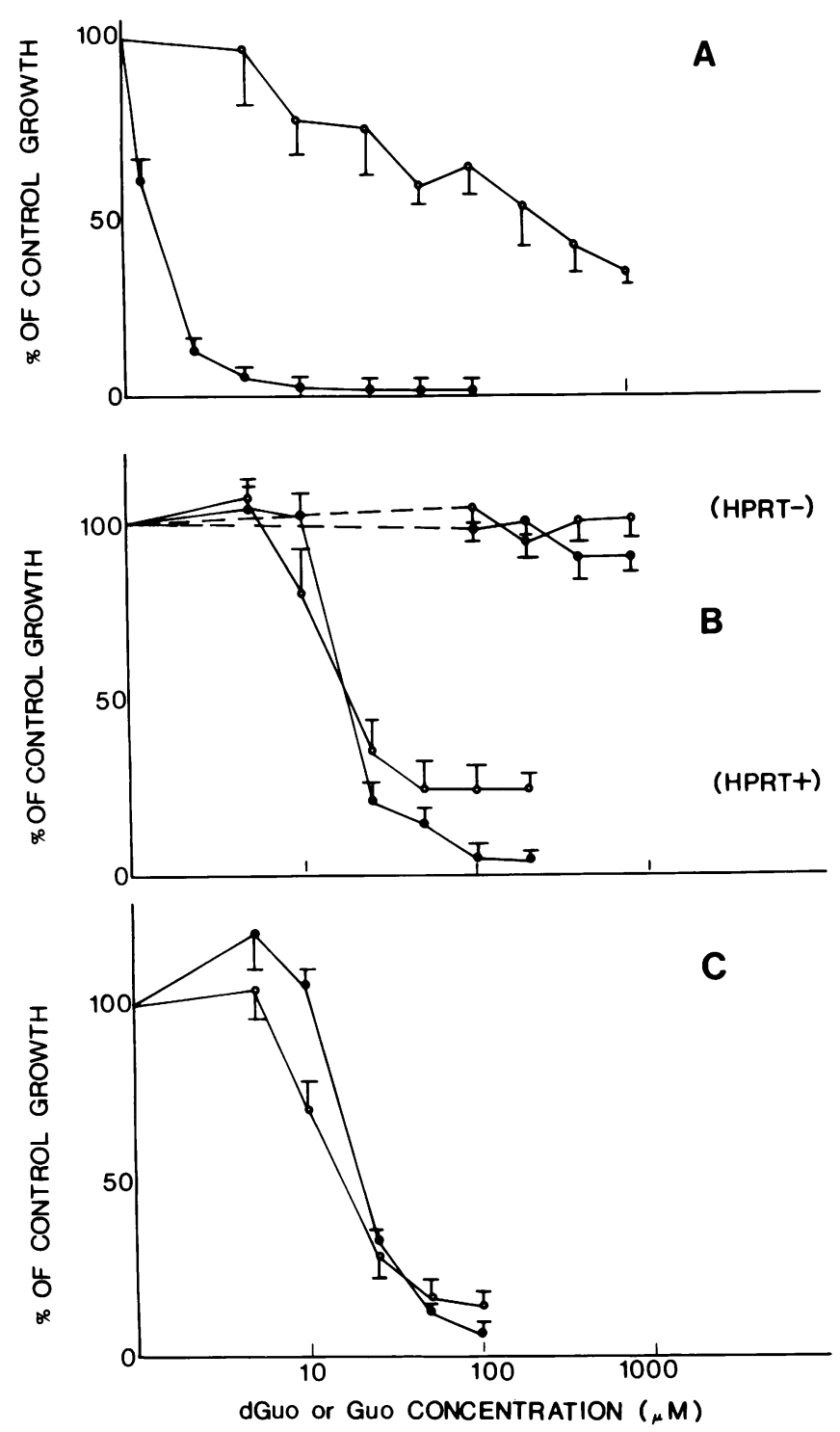

Figure 1. Relative effects of $2^{\prime}$-deoxyguanosine (dGuo) and guanosine (Guo) plus 8-aminoguanosine on the growth of $\mathrm{T}$ and $\mathrm{B}$ cell lines. (A) T lymphoblasts (Molt 4); (B) HPRT ${ }^{+}$B lymphoblasts (GM 558) and HPRT ${ }^{-}$B lymphoblasts (GM 1899); $(C)$ mature T cell line (HUT 78). Cells were grown in microtiter plates as described in Methods in the presence of the indicated concentrations of 2 '-deoxyguanosine (solid symbols) or guanosine (open symbols). The concentration of 8-aminoguanosine was $100 \mu \mathrm{M}$ in all experiments. Cell counts were performed after $72 \mathrm{~h}$. Values represent cell counts in the presence of additives relative to control cultures containing 8-aminoguanosine alone. 8-Aminoguanosine $(100 \mu \mathrm{M})$ alone had no effect on the growth of any cell line. Each point represents the mean $\pm S D$ of three experiments done in triplicate.

support this statement comes from the following observations: (a) HPRT-deficient B lymphoblasts which are incapable of the synthesis of guanine ribonucleotides from guanine are resistant
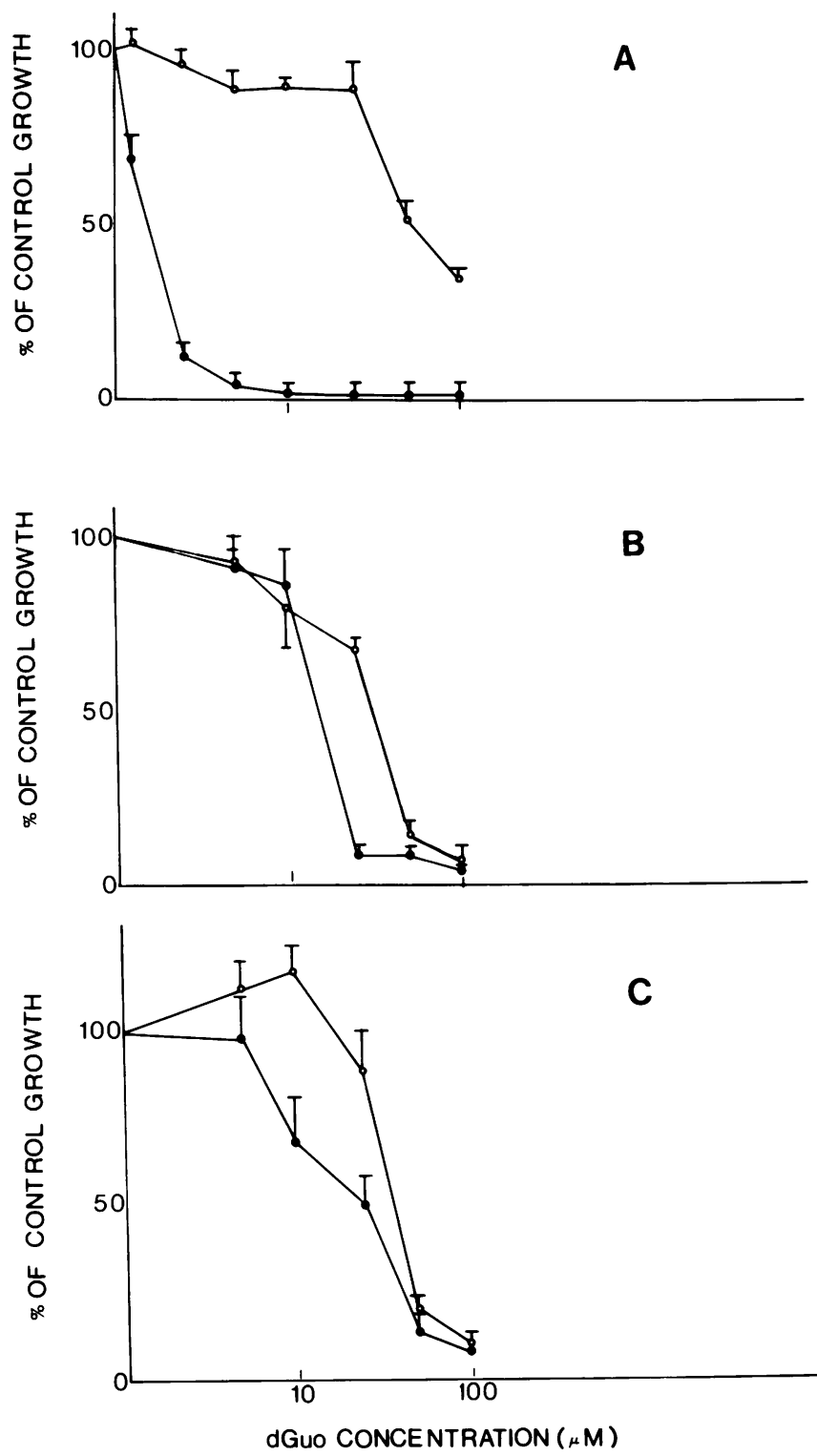

Figure 2. Effects of 2'-deoxycytidine on 2'-deoxyguanosine (dGuo) toxicity for T and B cell lines. $(A) \mathrm{T}$ lymphoblasts (Molt 4); $(B) \mathrm{B}$ lymphoblasts (GM 558); $(C)$ mature T cell line (HUT 78). Cells were grown in the presence of 2 -deoxyguanosine at the concentrations shown plus $100 \mu \mathrm{M} 8$-aminoguanosine in the absence $(-\bullet-)$ or presence $(-\circ-)$ of $100 \mu \mathrm{M} 2$-deoxycytidine. Values represent the mean $\pm S D$ of three experiments performed in duplicate.

to both GTP accumulation and 2'-deoxyguanosine or guanosine cytotoxicity; $(b)$ the addition of hypoxanthine or adenine rescues these cells and prevents GTP accumulation, presumably as a consequence of competing for and depleting PP-ribose-P pools; and $(c)$ incubation of these lymphoid cells with either guanosine or 2'-deoxyguanosine and 8-aminoguanosine leads to a three- to fivefold increase in GTP levels at $24 \mathrm{~h}$ which is 

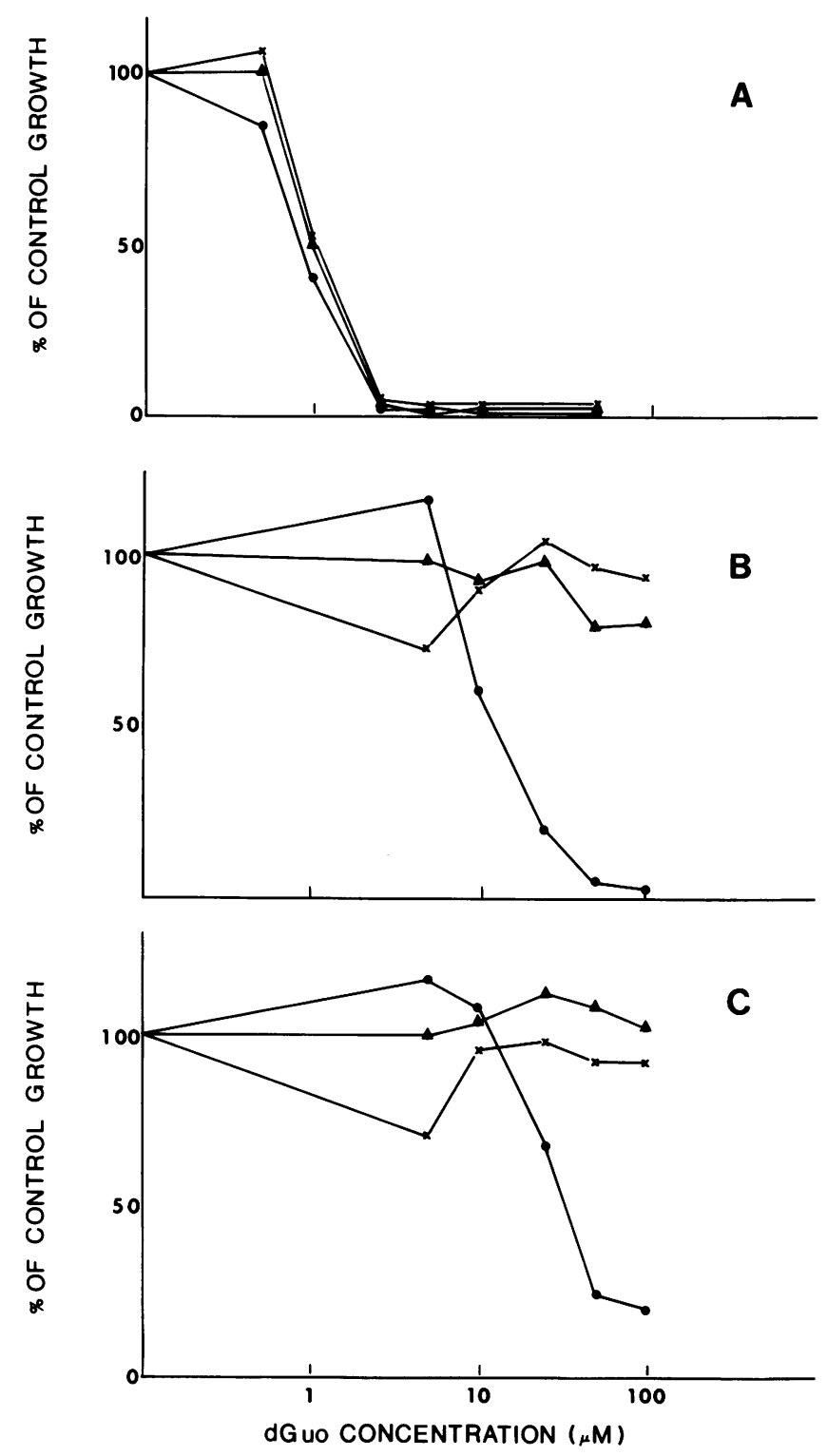

Figure 3. The effects of hypoxanthine or adenine on 2'-deoxyguanosine (dGuo) toxicity for $\mathrm{T}$ and $\mathrm{B}$ cell lines. $(A) \mathrm{T}$ lymphoblasts (Molt 4); (B) B lymphoblasts (GM 558); (C) mature T cell line (HUT 78). Cells were grown in the presence of 2'-deoxyguanosine at the concentrations shown plus $100 \mu \mathrm{M} 8$-aminoguanosine in the absence of adenine or hypoxanthine $(-\bullet-)$, in the presence of $100 \mu \mathrm{M}$ hypoxanthine $(-\times-)$, or in the presence of $100 \mu \mathrm{M}$ adenine $(-\Delta-)$. Each point represents the mean of triplicate determinations from a single experiment.

directly associated with a marked reduction in overall cell growth.

The evidence that the toxicity of 2 -deoxyguanosine for $T$ lymphoblasts is mediated by guanine deoxyribonucleotide rather than ribonucleotide accumulation is equally compelling: $(a)$ GTP levels fail to rise in T lymphoblasts in the face of large
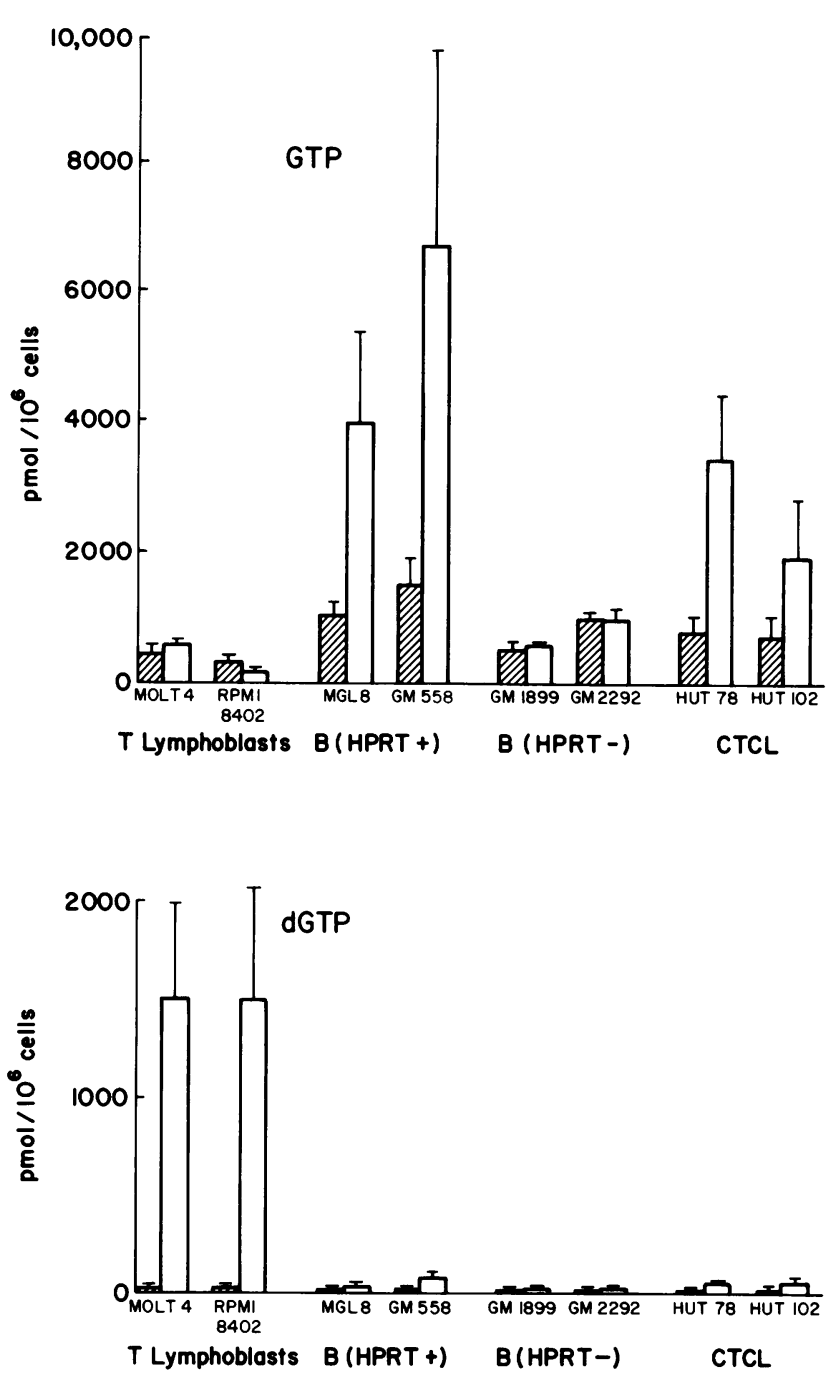

Figure 4. GTP and dGTP pools in T and B cell lines after incubations with 8-aminoguanosine with and without 2'-deoxyguanosine. Each bar represents the mean \pm SD of GTP or dGTP pools extracted from cells incubated for $24 \mathrm{~h}$ in the presence of $100 \mu \mathrm{M}$ 8-aminoguanosine alone (hatched bars) or $50 \mu \mathrm{M} 2$ '-deoxyguanosine plus 100 $\mu \mathrm{M}$ 8-aminoguanosine (open bars). Values were calculated from between three and nine separate experiments. B lymphoblast cell lines MGL 8 and GM 558 have normal HPRT activity (HPRT ${ }^{+}$), whereas lines GM 1899 and GM 2292 are deficient in this enzyme activity $\left(\right.$ HPRT $^{-}$). CTCL represents cutaneous $\mathrm{T}$ cell lymphoma cell lines of mature phenotype.

increases in dGTP; $(b)$ adenine and hypoxanthine have no salvaging effect; and (c) 2'-deoxycytidine rescues $\mathrm{T}$ lymphoblasts, but not $B$ lymphoblasts or mature $T$ cells, from 2'-deoxyguanosine toxicity. The rescue effect of $2^{\prime}$-deoxycytidine is presumed to result from the repletion of deoxycytidine triphosphate pools which are reduced as a consequence of dGTP accumulation and ribonucleotide reductase inhibition. In ad- 


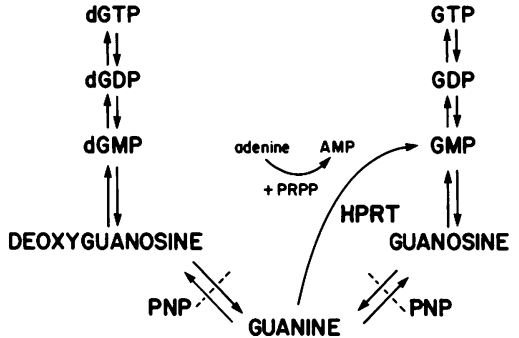

Figure 5. The metabolism of 2'-deoxyguanosine and guanosine. dition, 2'-deoxycytidine reduces the extent of 2'-deoxyguanosine phosphorylation and, hence, of dGTP accumulation by competing for deoxycytidine kinase activity (2). Thus, it is clear that the toxicity of 2'-deoxyguanosine for $B$ and mature $T$ cell lines is mediated by a completely different mechanism than that which has been previously established for $\mathrm{T}$ lymphoblasts. Differences between the metabolism of 2'-deoxyguanosine and guanosine by $\mathrm{B}$ and $\mathrm{T}$ lymphoblasts have also recently been reported by Osborne and Scott (14).

The role of 8-aminoguanosine as a potentiator of both 2 -deoxyguanosine and guanosine toxicity remains to be fully

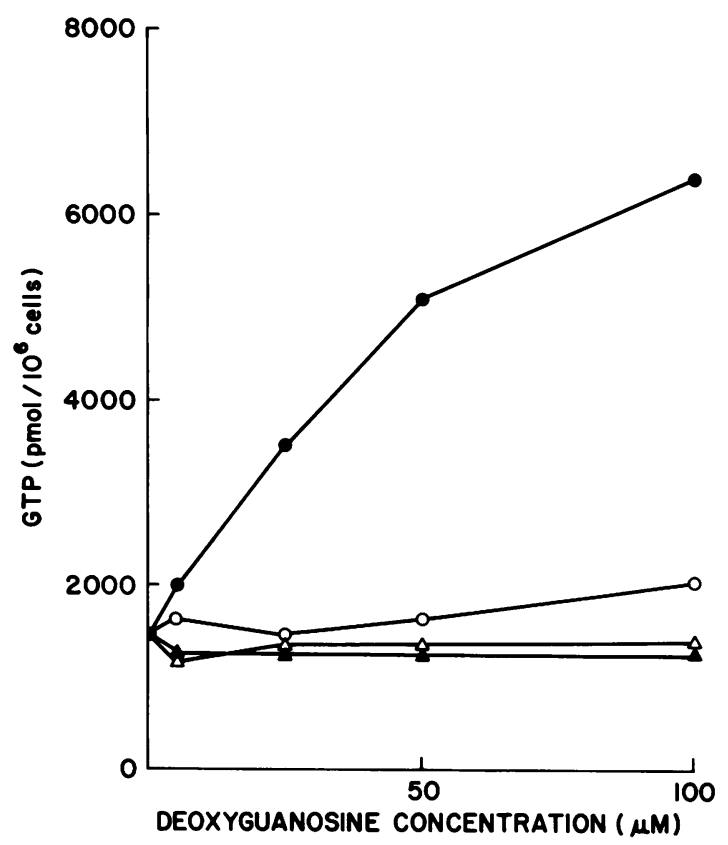

Figure 6. The effects of hypoxanthine and adenine on GTP accumulation in B lymphoblasts. GTP pools were measured in extracts of GM 558 cells after $24 \mathrm{~h}$ incubations with $50 \mu \mathrm{M} 2$ '-deoxyguanosine $(-\circ-), 50 \mu \mathrm{M} 2$-deoxyguanosine plus $100 \mu \mathrm{M}$ 8-aminoguanosine (-•-), $50 \mu \mathrm{M} 2$ '-deoxyguanosine plus $100 \mu \mathrm{M}$ 8-aminoguanosine plus $100 \mu \mathrm{M}$ hypoxanthine $(-\Delta-)$, or $50 \mu \mathrm{M}$ 2'-deoxyguanosine plus $100 \mu \mathrm{M}$ 8-aminoguanosine plus $100 \mu \mathrm{M}$ adenine $(-\Delta-)$. Values represent the results of a single representative experiment. Similar results were obtained with the HUT78 and MGL8 cell lines.
Table II. Comparative Effects of 2'-Deoxyguanosine, Guanosine, and Guanine on GTP Pools in B Lymphoblasts

\begin{tabular}{|c|c|c|c|c|c|c|}
\hline \multirow{2}{*}{$\begin{array}{l}\text { Concentration } \\
\text { of additive } \\
(\mu M)\end{array}$} & \multicolumn{2}{|c|}{ 2'-Deoxyguanosine } & \multicolumn{2}{|c|}{ Guanosine } & \multicolumn{2}{|l|}{ Guanine } \\
\hline & -8 AGuo & +8AGuo & $-8 \mathrm{AGuo}$ & +8AGuo & -8 AGuo & +8AGuo \\
\hline 10 & 1,538 & 1,990 & 1,711 & 1,411 & 1,615 & 1,043 \\
\hline 25 & 1,788 & 3,561 & 1,629 & 3,000 & 1,618 & 1,204 \\
\hline 50 & 1,776 & 5,078 & 1,803 & 4,321 & 1,690 & 1,254 \\
\hline 100 & 2,075 & 5,126 & 2,045 & 4,777 & 1,354 & 1,616 \\
\hline
\end{tabular}

GTP pools were measured in GM 558 B lymphoblasts which had been incubated for $24 \mathrm{~h}$ with the indicated additives in the absence or presence of 100 $\mu \mathrm{M}$ 8-aminoguanosine (8AGuo). Cells were extracted in $60 \%$ methanol and nucleotide pools determined by high pressure liquid chromatography, as described in Methods. Values are representative of those obtained in two additional experiments. GTP levels in the absence of any additives were $1673 \pm 345 \mathrm{pmol} / 10^{6}$ cells and in the presence of 8-aminoguanosine alone were $1525 \pm 390 \mathrm{pmol} / 10^{6}$ cells.

elucidated. This compound has been reported to be a relatively weak competitive inhibitor of PNP-catalyzed phosphorolysis of inosine with an inhibition constant $\left(K_{\mathrm{i}}\right)$ of $8 \mu \mathrm{M}(9,15)$. It has been demonstrated that 8 -aminoguanosine can be readily phosphorolyzed to 8-aminoguanine, also a competitive PNP inhibitor, by low amounts of PNP activity, which are equivalent to that present in horse serum, and that it prolongs the halflife of both guanosine and 2'-deoxyguanosine in tissue culture medium free of cells $(15,16)$. Our present experiments confirm that 8-aminoguanosine markedly prolongs the half-life of 2 deoxyguanosine in tissue culture medium containing cells, again presumably by inhibiting PNP activity. The prolongation in 2'-deoxyguanosine half-life correlates temporally with a far more prolonged accumulation of intracellular GTP. Paradoxically, however, both guanosine and 2'-deoxyguanosine would have to be cleaved to guanine by PNP to explain the complete resistance of HPRT-deficient B lymphoblasts to GTP accumulation. We, therefore, must postulate that 8-aminoguanosine does not completely inhibit intracellular PNP activity, but permits $2^{\prime}$-deoxyguanosine and guanosine to be transported intact into the cell where they are phosphorolyzed to guanine.

It is clear that guanine itself is less toxic to these lymphoid cell lines than is guanosine or 2 -deoxyguanosine in the presence of 8-aminoguanosine and does not cause prolonged elevations of GTP at $24 \mathrm{~h}$ at concentrations up to $100 \mu \mathrm{M}$. Two possible explanations for this differential effect must be considered. First, guanine could be less readily transported into the cell than are the guanine nucleosides. This appears unlikely since all available data indicate that the transport of guanine, analagous to that of hypoxanthine in cultured mammalian cells, is extremely rapid with uptake limited only by the rate of phosphoribosylation within the cell (17). Secondly, guanine could be rapidly depleted from the extracellular medium, making it unavailable for the sustained synthesis of GTP Although we were unable to measure guanine in the extracellular medium due to its loss during the deproteinization 

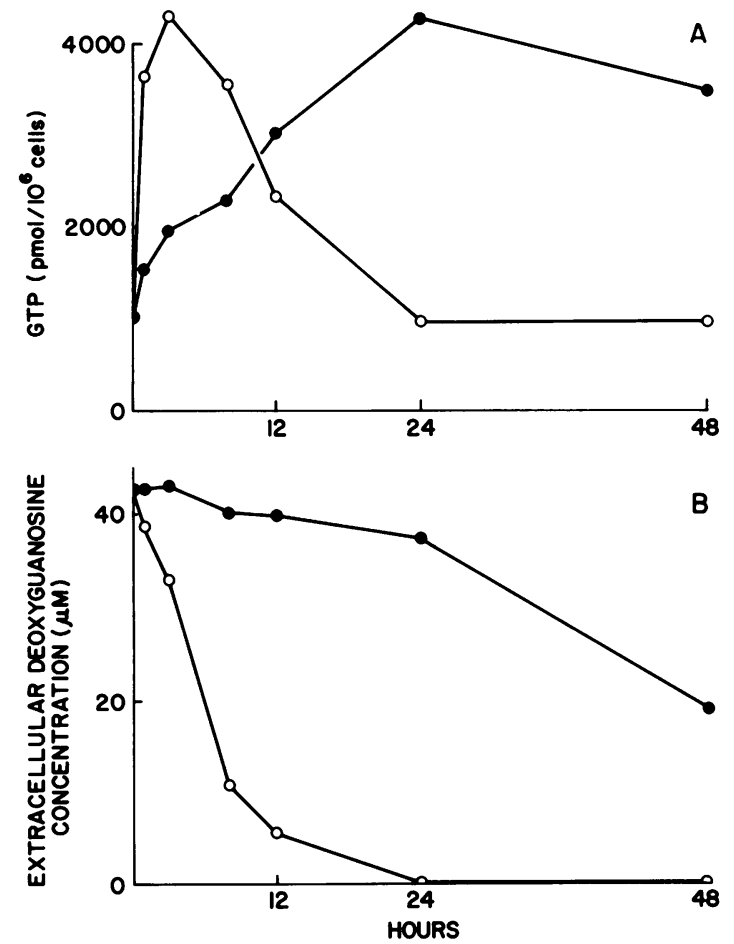

Figure 7. The effect of 8-aminoguanosine on extracellular 2'-deoxyguanosine concentrations and intracellular GTP pools in B lymphoblasts. Extracellular 2'-deoxyguanosine concentrations were measured in deproteinized supernates from cultures of MGL8 B lymphoblasts incubated in the presence of $50 \mu \mathrm{M} 2$-deoxyguanosine $(-\circ-)$ or $50 \mu \mathrm{M} 2$-deoxyguanosine plus $100 \mu \mathrm{M}$ 8-aminoguanosine $(-\bullet-)$ by high pressure liquid chromatography on a C-18 $\mu$ Bondapak reverse phase column, as described in Methods. Values are corrected for the $60 \%$ recovery of 2 -deoxyguanosine from the deproteinization procedure. GTP pools were measured in methanol extracts of cells sampled at the same time points. Values are those from a representative experiment; similar results were obtained in two additional experiments.

procedure, we favor this explanation. The addition of guanine to B lymphoblasts produced a very transient increase in GTP with a return to base-line levels by $5 \mathrm{~h}$. 8-Aminoguanosine did not prolong the time course of guanine-induced GTP accumulation. Therefore, it seems likely that guanine is transported into the cell and rapidly depleted from the medium in the process of generating guanine nucleotides over a short time period. We conclude from these observations that the prerequisite for guanine ribonucleotide accumulation which is suffcient to inhibit cell growth is the prolonged availability of intracellular guanine, leading to sustained elevations of intracellular guanine ribonucleotides.

Several previous studies with mutant cell lines have also implied that guanine ribonucleotide accumulation may have a role in inhibiting cell growth. Novikoff hepatoma cells selected for resistance to high concentrations of guanosine were found to be strikingly deficient in HPRT activity, indi-

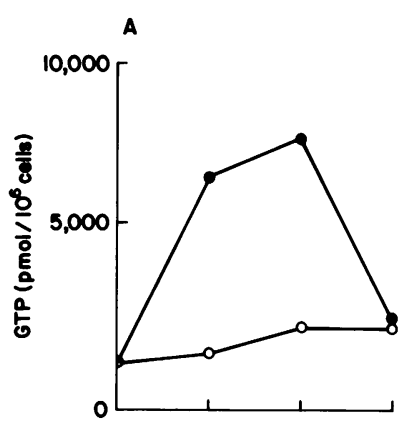

B

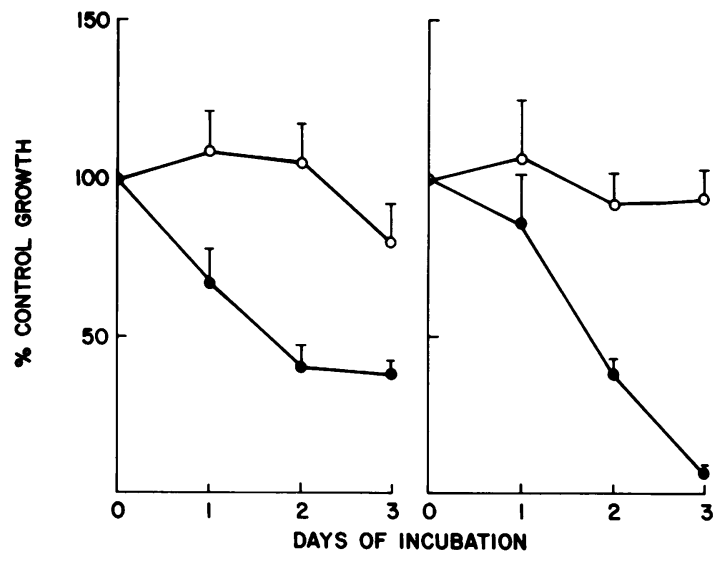

Figure 8. The effect of 8-aminoguanosine concentrations on GTP accumulation and cell growth. (A) B lymphoblasts (GM 558); (B) mature T cell line (HUT 78). Cells were grown in the presence of 50 $\mu \mathrm{M} 2$-deoxyguanosine and $100 \mu \mathrm{M}(-\bullet-)$ or $400 \mu \mathrm{M}$ (-o-) 8-aminoguanosine. GTP values represent the mean of two experiments and the cell growth data the mean $\pm S D$ of five determinations.

cating that nucleotide synthesis was important in mediating the sensitivity of wild type cells to guanosine (18). Guanine and guanosine in $100 \mu \mathrm{M}$ concentrations have also been reported to inhibit the growth of human lymphoblasts in association with GTP accumulation, while HPRT-deficient cells were unaffected (19). In addition, Chinese hamster ovary cells and mouse $\mathrm{T}$ lymphoma cells deficient in the enzyme adenylosuccinate synthetase, an enzyme which is rate limiting for the conversion of inosine monophosphate into the adenylate nucleotide pool, have been shown to be highly sensitive to both guanine and guanosine $(20,21)$. Although the effects of exogenous nucleosides on nucleotide pools were not measured, the mutant $\mathrm{T}$ lymphoma line contained three times more GTP and GMP than did the wild type cells. It was implied that the block in adenine nucleotide synthesis facilitated the accumulation of guanine nucleotides within these cells and that growth inhibition resulted from the imbalance between the two pools. Finally, mouse $T$ lymphoma cells deficient in both HPRT and deoxycytidine kinase are more resistant to 2 '-deoxyguanosine toxicity than are mutants deficient in deoxycytidine kinase alone (22), while 2'-deoxyguanosine toxicity for these cells is more completely reversed by the addition of 
both 2 -deoxycytidine and hypoxanthine than by 2 -deoxycytidine alone (23). Again, these data support the importance of guanine salvage as one component of the cytotoxic effect of 2'-deoxyguanosine.

Although we have measured only the nucleoside triphosphate pools in these experiments, it is clear that elevation of GTP levels may be associated with concomitant increases in GMP and guanosine diphosphate (GDP). The mechanism whereby one or more of these guanine ribonucleotides inhibit growth remains a subject for some speculation. One major possibility is that they inhibit de novo purine biosynthesis, with consequent depletion of the adenine nucleotide pools. The exact mechanism by which guanine nucleotides inhibit purine biosynthesis in intact cells in unclear. Ullman et al. observed an increase in PP-ribose-P pools and a threefold reduction in purine synthetic rates in their adenylosuccinate synthetase-deficient cells and concluded that guanine nucleotides inhibit glutamine PP-ribose-P-amidotransferase, which catalyzes the first reaction specifically concerned with purine biosynthesis (20). This enzyme is known to be allosterically regulated by a number of purine ribonucleotides including GMP and, to a lesser extent, GDP and GTP $(24,25)$. However, others have been unable to demonstrate inhibition of this enzyme under conditions yielding a threefold increase in GTP levels by guanine and inhibition of purine biosynthesis (26). Depletion of PP-ribose-P by guanine appears an unlikely mechanism, since the addition of hypoxanthine or adenine, which effectively reduced PP-ribose-P levels to the point of preventing guanine ribonucleotide synthesis, salvaged the cells rather than producing any growth inhibitory effects. Finally, pyrimidine starvation can be excluded since the addition of uridine did not rescue the cells.

Although the mechanism remains somewhat uncertain, the observation that a competitive inhibitor of PNP can potentiate the toxicity of 2'-deoxyguanosine and guanosine for lymphoid cells other than $\mathrm{T}$ lymphoblasts has important implications for the use of such agents as immunosuppressive drugs. Inherited PNP deficiency is known to result in the overproduction of all four PNP substrates-inosine, 2'-deoxyinosine, guanosine, and 2 '-deoxyguanosine (27). It is clear that the use of enzyme inhibitors in vivo can lead to increases in substrate concentrations to levels which far surpass those found in the congenital deficiency state (28). Thus, the pharmacologic use of PNP inhibitors which are not tight-binding inhibitors and/ or which result in only partial inhibition of intracellular PNP activity might well be associated with significant and prolonged elevation of both guanosine and 2'-deoxyguanosine concentrations in the plasma and with guanine ribonucleotide accumulation which is not limited to the target $T$ cell population. In addition, the observation that $T$ lymphoblasts remain about 10-fold more sensitive to 2'-deoxyguanosine plus 8-aminoguanosine than do mature $T$ cell lines raises the question as to whether ablation of differentiated $\mathrm{T}$ cells can be achieved in the absence of more generalized guanine ribonucleotide accumulation and nonspecific cellular toxicity.
These alternate metabolic effects do not, however, appear to play a role in inherited PNP-deficiency disease. In fact, erythrocytes from a PNP-deficient child have been found to have very low levels of GTP, while peripheral blood lymphocyte GTP levels are apparently within the normal range (29). It remains possible that a selective depletion of immature $T$ cells can be achieved by more potent PNP inhibitors than are currently available.

Note added in proof. Subsequent to the submission of this work, Spaapen et al. have demonstrated that guanine ribonucleotide accumulation contributes to inhibition of mitogenic response in peripheral blood lymphocytes cultured with guanosine or 2'-deoxyguanosine plus 8-aminoguanosine (30).

\section{Acknowledgments}

We thank Jerrie CeBulkey for her help with the manuscript.

This work was supported by grant CA 34085 awarded by the National Cancer Institute, Department of Health and Human Services. Dr. Sidi is the recipient of an Elsa Pardee Fellowship from the University of Michigan. Dr. Mitchell is the recipient of a Scholar Award from the Leukemia Society of America.

\section{References}

1. Giblett, E. R., A. J. Ammann, R. S. Sandman, D. W. Wara, and L. K. Diamond. 1975. Nucleoside phosphorylase deficiency in a child with severely defective T-cell immunity and normal B-cell immunity. Lancet. I:1010-1013.

2. Martin, D. W., Jr., and E. Gelfand. 1981. Biochemistry of diseases of immunodevelopment. Annu. Rev. Biochem. 50:845-877.

3. Cohen, A., L. J. Gudas, A. J. Ammann, G. E. J. Staal, and D. W. Martin, Jr. 1978. Deoxyguanosine triphosphate as a possible toxic metabolite in the immunodeficiency associated with purine nucleoside phosphorylase deficiency. J. Clin. Invest. 61:1405-1409.

4. Mitchell, B. S., E. Mejias, P. E. Daddona, and W. N. Kelley. 1978. Purinogenic immunodeficiency diseases. Selective toxicity of deoxyribonucleosides for T cells. Proc. Natl. Acad. Sci. USA. 75:50115014.

5. Carson, D. A., J. Kaye, S. Matsumoto, J. E. Seegmiller, and L. Thompson. 1979. Biochemical basis for the enhanced toxicity of deoxyribonucleosides toward malignant human T cell lines. Proc. Natl. Acad. Sci. USA. 76:2430-2433.

6. Wilson, J. M., B. S. Mitchell, P. E. Daddona, and W. N. Kelley. 1979. Purinogenic immunodeficiency diseases. Differential effects of deoxyadenosine and deoxyguanosine on DNA synthesis in human $T$ lymphoblasts. J. Clin. Invest. 64:1475-1484.

7. Ullman, B., S. M. Clift, L. J. Gudas, B. B. Levinson, M. A. Wormsted, and D. W. Martin, Jr. 1980. Alterations in deoxyribonucleotide metabolism in cultured cells with ribonucleotide reductase activities refractory to feedback inhibition by 2 '-deoxyadenosine triphosphate. J. Biol. Chem. 255:8308-8314.

8. Carson, D. A., D. B. Wasson, E. Lakow, and N. Kamatani. 1982. Possible metabolic basis for the different immunodeficient states associated with genetic deficiencies of adenosine deaminase and purine nucleoside phosphorylase. Proc. Natl. Acad. Sci. USA. 79:3848-3852.

9. Kazmers, I. S., B. S. Mitchell, P. E. Daddona, L. L. Wotring, L. B. Townsend, and W. N. Kelley. 1981. Inhibition of purine 
nucleoside phosphorylase by 8 -aminoguanosine. Selective toxicity for T lymphoblasts. Science (Wash. DC). 214:1137-1139.

10. Cohen, A., W. W. Jacob, H. M. Dosch, and E. W. Gelfand. 1980. The expression of deoxyguanosine toxicity in $T$ lymphocytes at different stages of maturation. J. Immunol. 125:1578-1582.

11. Gazdar, A. F., D. N. Carney, P. A. Bunn, E. K. Russell, E. S. Jaffe, G. P. Schlechter, and L. G. Guccion. 1980. Mitogen requirements for the in vitro propagation of cutaneous T-cell lymphoma. Blood. 55:409-417.

12. Poiesz, B. J., F. W. Ruscetti, A. F. Gazdar, P. A. Bunn, J. D. Minna, and R. C. Gallo. 1980. Detection and isolation of type C retrovirus particles from fresh and cultured lymphocytes of a patient with cutaneous T-cell lymphoma. Proc. Natl. Acad. Sci. USA. 77:74157419.

13. Koller, C. A., P. L. Stetson, L. D. Nichamin, and B. S. Mitchell. 1980. An assay of adenosine and deoxyadenosine in human plasma by HPLC. Biochem. Med. 24:179-184.

14. Osborne, W. R. A., and C. R. Scott. 1983. The metabolism of deoxyguanosine and guanosine in human $B$ and $T$ lymphoblasts. Biochem. J. 214:711-718.

15. Stoeckler, J. D., C. Cambor, V. Kuhns, S. H. Chu, and R. F. Parks, Jr. 1982. Inhibitors of purine nucleoside phosphorylase, C(8) and $C\left(5^{\prime}\right)$ substitutions. Biochem. Pharmacol. 31:163-171.

16. Harden, R. A., T. K. Huard, and B. S. Mitchell. 1983. Relative effects of purine nucleoside phosphorylase inhibitors and guanosine analogues in mitogen-induced T lymphocyte proliferation. Clin. Res. 31:344A.

17. Marz, R., R. M. Wohlhueter, and P. G. W. Plagemann. 1979. Purine and pyrimidine transport and phosphoribosylation and their interaction in overall uptake by cultured mammalian cells: a reevaluation. J. Biol. Chem. 254:2329-2338.

18. May, R. A., and P. Hoffee. 1978. Guanosine metabolism in Novikoff hepatoma cells: isolation and characterization of guanosine resistant variants. Arch. Biochem. Biophys. 190:712-719.

19. Brenton, D. P., K. H. Astrin, M. K. Cruikshank, and J. E. Seegmiller. 1977. Measurement of free nucleotides in cultured human lymphoid cells using high pressure liquid chromatography. Biochem. Med. 17:231-247.

20. Ullman, B., S. M. Clift, A. Cohen, L. J. Gudas, B. B. Levinson,
M. A. Wormsted, and D. W. Martin, Jr. 1979. Abnormal regulation of de novo purine synthesis and purine salvage in a cultured mouse T-cell lymphoma mutant partially deficient in adenylosuccinate synthetase. J. Cell Physiol. 99:139-151.

21. Tu, A. S., and D. Patterson. 1978. Characterization of a guanine-sensitive mutant defective in adenylosuccinate synthetase activity. J. Cell Physiol. 96:123-132.

22. Gudas, L. J., B. Ullman, A. Cohen, and D. W. Martin, Jr. 1978. Deoxyguanosine toxicity in a mouse $T$ lymphoma. Relationship to purine nucleoside phosphorylase associated immune dysfunction. Cell. 14:531-538.

23. Chan, T. S. 1978. Deoxyguanosine toxicity on lymphoid cells as a cause for immunosuppression in purine nucleoside phosphorylase deficiency. Cell. 14:523-530.

24. Itakura, M., R. L. Sabina, P. W. Heald, and E. W. Holmes. 1981. Basis for the control of purine biosynthesis by purine ribonucleotides. J. Clin. Invest. 67:994-1002.

25. Holmes, E. W., J. A. McDonald, J. M. McCord, M. B. Wyngaarden, and W. N. Kelley. 1973. Human glutamine phosphoribosyl pyrophosphate amidotransferase: kinetic and regulatory properties. $J$. Biol. Chem. 248:144-150.

26. Bagnara, A. S., A. A. Letter, and J. F. Henderson. 1974. Multiple mechanisms of regulation of purine biosynthesis de novo in intact tumor cells. Biochim. Biophys. Acta. 374:259-270.

27. Cohen, A., D. Doyle, D. W. Martin, Jr., and A. J. Ammann. 1976. Abnormal purine metabolism and purine overproduction in a patient deficient in purine nucleoside phosphorylase. N. Engl. J. Med. 295:1449-1454.

28. Mitchell, B. S. 1982. Deficiencies of adenosine deaminase and purine nucleoside phosphorylase: implications for immunopharmacology. Med. Gr. Rounds. 1:85-93.

29. Simmonds, H. A., A. R. Watson, D. R. Webster, A. Sahota, and D. Perrett. 1982. GTP depletion and other erythrocyte abnormalities in inherited PNP deficiency. Biochem. Pharmacol. 31:941-946.

30. Spaapen, L. J. M., G. T. Rijkers, G. E. J. Staal, G. Rijksen, S. K. Wadman, J. W. Stoop, and B. J. M. Zegers. 1984. The effect of deoxyguanosine on human lymphocyte function. J. Immunol. 132:23112323. 